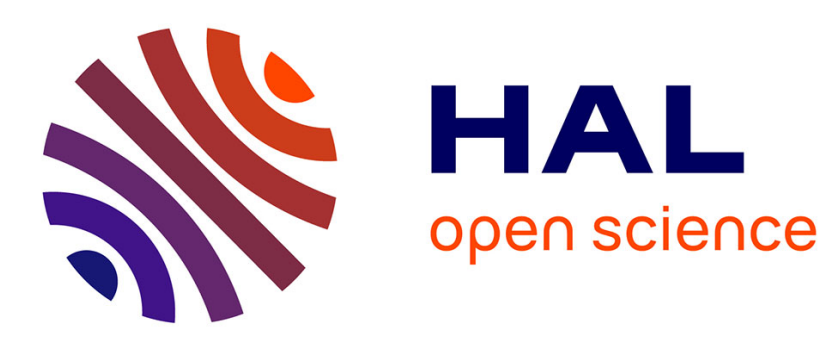

\title{
A simplified proof of a Liouville theorem for nonnegative solution of a subcritical semilinear heat equations \\ Nejla Nouaili
}

\section{To cite this version:}

Nejla Nouaili. A simplified proof of a Liouville theorem for nonnegative solution of a subcritical semilinear heat equations. Journal of Dynamics and Differential Equations, 2009, 21 (1), pp.127-132. 10.1007/s10884-008-9121-6 . hal-00701234

\section{HAL Id: hal-00701234 \\ https://hal.science/hal-00701234}

Submitted on 24 May 2012

HAL is a multi-disciplinary open access archive for the deposit and dissemination of scientific research documents, whether they are published or not. The documents may come from teaching and research institutions in France or abroad, or from public or private research centers.
L'archive ouverte pluridisciplinaire HAL, est destinée au dépôt et à la diffusion de documents scientifiques de niveau recherche, publiés ou non, émanant des établissements d'enseignement et de recherche français ou étrangers, des laboratoires publics ou privés. 


\title{
A simplified proof of a Liouville theorem for nonnegative solution of a subcritical semilinear heat equations
}

\author{
Nejla Nouaili \\ Ecole Normale Supérieure and Ecole Polytechnique de Tunisie.
}

June 12, 2008

\begin{abstract}
We give a new proof of the Liouville theorem proved by Merle and Zaag for nonnegative solutions of the semilinear heat equation with power nonlinearity. Our proof has a pedagogical interest and is based on Kaplan's blow-up criterion.
\end{abstract}

Mathematical Subject classification: 35K05, 35K55, 35A20.

Keywords: heat equation, Liouville theorem, blow-up, Kaplan's criterion.

\section{Introduction}

In [MZ98] and [MZ00], Merle and Zaag consider the following semilinear heat equation

$$
u_{t}=\Delta u+|u|^{p-1} u
$$

and prove the following Liouville theorem:

Theorem 1(Merle-Zaag) Assume that

$$
p>1 \text { and }(N-2) p<N+2 .
$$

Consider $u$ a solution of (1) defined for all $(x, t) \in \mathbb{R}^{N} \times(-\infty, T)$. Assume in addition that $|u(x, t)| \leq C(T-t)^{-\frac{1}{p-1}}$, for some constant $C>0$. Then $u \equiv 0$ or there exists $T_{0} \geq T$ such that for all $(x, t) \in \mathbb{R}^{N} \times(-\infty, T), u(x, t)= \pm \kappa\left(T_{0}-t\right)^{-\frac{1}{p-1}}$ with $\kappa=(p-1)^{-\frac{1}{p-1}}$.

Introducing the following similarity variables:

$$
y=\frac{x}{\sqrt{T-t}}, s=-\log (T-t), w(y, s)=(T-t)^{\frac{1}{p-1}} u(x, t),
$$

equation (1) is transformed in the following equation:

$$
w_{s}=\Delta w-\frac{1}{2} y \cdot \nabla w-\frac{1}{p-1} w+|w|^{p-1} w
$$


and we get another equivalent formulation of the above Liouville theorem.

Theorem 1'(Merle-Zaag) Assume (2) and consider $w(y, s)$ a bounded solution of (4), defined for all $(y, s) \in \mathbb{R}^{N} \times \mathbb{R}$. Then $w$ is one of the following solutions: $w \equiv 0$, or $w= \pm \kappa$, or there exist $s_{0} \in \mathbb{R}$, such that $w= \pm \varphi\left(s-s_{0}\right)$ with $\varphi(s)=\kappa\left(1+e^{s}\right)^{-\frac{1}{p-1}}$.

This theorem introduces a new approach in the study of equation (1), in the sense that it gives uniform estimates both in space and with respect to initial data. For instance, the following localization property is proved in [MZ98] and [MZ00]:

Uniform ODE Behavior: Consider $T \leq T_{0},\left\|u_{0}\right\|_{C^{2}\left(\mathbb{R}^{N}\right)} \leq C_{0}$ and $u(x, t)$ the solution of equation (1), with initial data $u_{0}$. Then, for all $\epsilon>0$, there is $C\left(\epsilon, C_{0}, T_{0}\right)$ such that for all $x \in \mathbb{R}^{N}$ and $t \in[0, T)$,

$$
\left.\left.\left|\frac{\partial u}{\partial t}(x, t)-\right| u\right|^{p-1} u(x, t)|\leq \epsilon| u(x, t)\right|^{p}+C .
$$

The above uniform estimate allowed to get new blow-up results for equation (1), unknown before, such as the stability of the blow up profile (see Fermanian, Merle and Zaag [FKMZ00]) and the regularity of the blow-up set (see Zaag [Zaa06]).

Moreover, the approach consisting in proving Liouville theorems in order to get new blowup results has been successful for other parabolic equations with no gradient structure (see Nouaili and Zaag [NZ07]), hyperbolic equations like Korteweg de Vries (Martel and Merle [MM00]) and the wave equation (Merle and Zaag [MZ08]).

In [MZ98] and [MZ00], the authors prove the Liouville theorem in similarity variables. They use the following Lyapunov functional associated with (4):

$$
E(w)=\int_{\mathbb{R}^{N}}\left(\frac{1}{2}|\nabla w|^{2}+\frac{|w|^{2}}{2(p-1)}-\frac{|w|^{p+1}}{p+1}\right) \rho d y, \text { where } \rho(y)=\frac{e^{-\frac{|y|^{2}}{4}}}{(4 \pi)^{N / 2}} .
$$

The heart of the proof is the linearization of $w$ (defined by (4)) around $\kappa$ as $s \rightarrow-\infty$. Using similar ideas to Filippas and Kohn [FK92], Merle and Zaag prove that there are at most three possible ways in which $w$ goes to $\kappa$ as $s \rightarrow-\infty$. Then, they show that one of the three cases corresponds to $w(y, s)=\varphi\left(s-s_{0}\right)$ for some $s_{0} \in \mathbb{R}$ where $\varphi(s)=\kappa\left(1+e^{s}\right)^{-\frac{1}{p-1}}$. In the other two cases, they find a contradiction using the following blow-up criterion:

Let $w$ be a solution of (4) which satisfies $I\left(w\left(s_{0}\right)\right)>0$ for some $s_{0} \in \mathbb{R}$ where

$$
I(w(s))=-2 E(w(s))+\frac{p-1}{p+1}\left(\int_{\mathbb{R}^{N}}|w(y, s)|^{2} \rho(y) d y\right)^{\frac{p+1}{2}} .
$$

Then, $w$ blows up at some time $S>s_{0}$.

In this note, we found that in the nonnegative case (treated in [MZ98]), we could avoid the long and technical linearization around $\kappa$ as $s \rightarrow-\infty$ and the application of the blow-up criterion. More precisely, we found that the Liouville theorem in the nonnegative 
case follows from Kaplan's blow-up criterion for equation (1) (see [Kap63]) and the work of Giga and Kohn (see [GK85], [GK87] and [GK89]), which are more simple ingredients. The aim of this note is to present this more simple proof, which is pedagogically easier then the analysis of [MZ98]. Of course, for unsigned solutions, we cannot escape the proof given in [MZ00], which heavily relies on the preceding paper [MZ98].

We proceed in two sections:

In section 2, for the reader's convenience, we give and prove our version of Kaplan's criterion, making our paper more self contained.

In section 3, we give our proof of the Liouville theorem in the nonnegative case.

Acknowledgment: We would like to thank the referee for his careful reading and helpful remarks. He suggests the use of Kaplan's blow-up criterion instead of Fujita's, which we used in the first version of this paper.

\section{Kaplan's blow-up criterion}

In the following, we give our version of Kaplan's criterion:

Proposition 2.1 (Kaplan's blow-up criterion for equation (1)) There exists $M>0$ such that if $V_{0}(x)$ in $L^{\infty}\left(\mathbb{R}^{N}\right)$ is nonnegative and satisfies

$$
\forall|x| \leq M, \quad V_{0}(x) \geq \frac{\kappa}{2}
$$

then the solution $V(x, t)$ of equation (1) with initial data $V_{0}$ blows up in finite time $T>0$.

Proof: Here we use Kaplan's method introduced in [Kap63] (see Theorem 8 page 327). Note that since $V_{0}(x)$ is nonnegative, the same holds for $V(x, t)$ whenever it exists.

We note by $\lambda>0$ the first eigenvalue of $-\Delta$ on the ball $B(0, M)$ and by $\psi(x)$ the corresponding eigenfunction to $\lambda$. In other words, we have

$$
\begin{cases}-\Delta \psi & =\lambda \psi \text { in } B(0, M) \\ \psi & =0 \text { on } \partial B(0, M) .\end{cases}
$$

Note that $\psi(x) \geq 0$ in $B(0, M)$ and from scaling arguments, we have $\lambda=\frac{\lambda_{1}}{M^{2}}$, where $\lambda_{1}>0$ is the first eigenvalue of $-\Delta$ on the ball $B(0,1)$. We also assume that $\int_{B(0, M)} \psi(x) d x=1$. We define $\hat{V}(t)=\int_{B(0, M)} V(x, t) \psi(x) d x$. Multiplying both sides of (1) by $\psi(x)$ and integrating over $B(0, M)$, then using Jensen's inequality and integration by parts, we obtain

$$
\left\{\begin{array}{l}
\hat{V}^{\prime}(t) \geq-\lambda_{1} \hat{V}+\hat{V}(t)^{p} \text { wherever } V \text { is defined } \\
\hat{V}(0) \geq \frac{\kappa}{2}
\end{array}\right.
$$

(see [Kap63] for details).

Now, we note by $\Phi(t)$ the solution of the ODE:

$$
\left\{\begin{array}{l}
\Phi^{\prime}(t)=\Phi(t)^{p}-\lambda \Phi(t) \\
\Phi(0)=\frac{\kappa}{2}
\end{array}\right.
$$

Since $\lambda=\frac{\lambda_{1}}{M^{2}}$, we can see that taking $M$ large enough, $\Phi(t)$ blows up at some finite time $T_{0}>0$, hence by $(6), \hat{V}(t) \geq \Phi(t)$ and $\hat{V}$ blows up at some earlier time.

Using the fact that $\sup _{x \in \mathbb{R}^{N}} V(x, t) \geq \hat{V}(t)$, we conclude that $V(x, t)$ blows up in finite time $T>0$. 


\section{Our new proof of the Liouville Theorem}

We consider $w$ a nonnegative, global and bounded solution of (4). We proceed in two steps. First, we find limits $w_{ \pm \infty}$ for $w$ as $s \rightarrow \pm \infty$ and reach a conclusion in some trivial cases. In a second step, we focus on the case where $w_{-\infty}=\kappa$ as $s \rightarrow-\infty$ and conclude the proof.

\section{Step 1: Limits of $w$ as $s \rightarrow \pm \infty$}

We recall respectively, Theorem 1 and Proposition 5 from [GK85] (page 305 and 309), under the condition (2).

Proposition 3.1 (Stationary problem of (4) (Giga-Kohn)) The only global solutions in $L^{\infty}\left(\mathbb{R}^{N}\right)$ of

$$
0=\Delta w-\frac{1}{2} y \cdot \nabla w-\frac{w}{p-1}+|w|^{p-1} w,
$$

are the constant ones $w \equiv 0, w \equiv-\kappa$ and $w \equiv \kappa$.

Proposition 3.2 (Limits of $w$ as $s \rightarrow \pm \infty$ (Giga-Kohn)) Let $w$ be a bounded global solution of (4) in $\mathbb{R}^{N}$. Then, $\lim _{s \rightarrow \infty} w(y, s)$ exists and equals 0 or $\pm \kappa$. The convergence takes place in $C^{2}(B(0, R))$ for any $R>0$. The corresponding statements holds also for the limits as $s \rightarrow-\infty$.

From the propositions above and the positivity of $w$, we have $w_{ \pm \infty} \equiv 0$ or $w_{ \pm \infty} \equiv \kappa$. Since $E$ is a Lyapunov functional for $w$, one gets from (4) and (5):

$$
0 \leq \int_{-\infty}^{+\infty} d s \int_{\mathbb{R}^{N}}\left|\frac{\partial w}{\partial s}(y, s)\right|^{2} \rho d y=E\left(w_{-\infty}\right)-E\left(w_{+\infty}\right) .
$$

Therefore, since $E(\kappa)>0=E(0)$, there are only two cases:

- $E\left(w_{-\infty}\right)=E\left(w_{+\infty}\right)$. This implies that $\frac{\partial w}{\partial s} \equiv 0$, hence $w$ is a stationary solution of (4) and $w \equiv 0$ or $w \equiv \kappa$ by Proposition 3.1.

- $E\left(w_{-\infty}\right)-E\left(w_{+\infty}\right)>0$. This occurs only if $w_{+\infty} \equiv 0$ and $w_{-\infty} \equiv \kappa$. It remains to treat this case:

Step 2: Case where $w \rightarrow \kappa$ as $s \rightarrow-\infty$

Consider $M>0$ given by Proposition 2.1. From Proposition 3.2, there is some time $s^{*}$ negative and large enough such that

$$
w\left(y, s^{*}\right) \geq \frac{\kappa}{2} \text { for all }|y|<M .
$$

Introducing $v(x, t)$ defined by

$$
v(x, t)=(1-t)^{-\frac{1}{p-1}} w\left(y, s+s^{*}\right) \text { where } y=\frac{x}{\sqrt{1-t}} \text { and } s=-\log (1-t),
$$


we see that the function $v$ is defined for all $x \in \mathbb{R}^{N}$ and $t<1$, satisfies (1) and

$$
\forall x \in \mathbb{R}^{N}, \quad v(x, 0)=w\left(y, s^{*}\right) .
$$

If we consider $V(x, t)$ the solution of (1) with initial condition $V(x, 0)=v(x, 0)$, then from (8), (10) and Proposition 2.1, we have that $V$ blows up at some finite time $T>0$.

Since we have from uniqueness for (1) that

$$
\forall(x, t) \in \mathbb{R}^{N} \times[0,1), \quad V(x, t)=v(x, t),
$$

this gives $T \geq 1$. Extending $v(x, t)$ for $t \geq 1$ (if ever $T>1$ ) by $v(x, t)=V(x, t)$, we see that $v(x, t)$ is a solution of $(1)$ defined for all $(x, t) \in \mathbb{R}^{N} \times(-\infty, T)$ and which blows up at time $T \geq 1$ (note in particular that (9) still holds)

Now, if we consider $a \in \mathbb{R}^{N}$ a blow-up point of $v$ and introduce the following similarity variables:

$$
y^{\prime}=\frac{x-a}{\sqrt{T-t}}, s^{\prime}=-\log (T-t), w_{a}\left(y^{\prime}, s^{\prime}\right)=(T-t)^{\frac{1}{p-1}} v(x, t),
$$

then, we see from Giga and Kohn [GK87], Theorem 3.7 (page 17) that:

$$
\forall s^{\prime} \in \mathbb{R},\left\|w_{a}\left(s^{\prime}\right)\right\|_{L^{\infty}} \leq C_{1} \text {, where } C_{1}>0,
$$

and from [GK89], Corollary 3.4 (page 872), that:

$$
w_{a}\left(y^{\prime}, s^{\prime}\right) \rightarrow \kappa \text { as } s^{\prime} \rightarrow+\infty \text {, uniformly on compact sets. }
$$

In the following, we are looking for the limit of $w_{a}$ as $s^{\prime} \rightarrow-\infty$. Using (9) and (11), we obtain:

$$
\begin{aligned}
& w_{a}\left(y^{\prime}, s^{\prime}\right)=(1-\sigma)^{-\frac{1}{p-1}} w(y, s) \text { where } \sigma=(T-1) e^{s^{\prime}}, \\
& y=\frac{y^{\prime}+a e^{s^{\prime} / 2}}{\sqrt{1-\sigma}} \text { and } s=s^{\prime}-\log (1-\sigma)+s^{*} .
\end{aligned}
$$

Since $w(y, s) \rightarrow \kappa$ as $s \rightarrow-\infty$ uniformly on compact sets and $\sigma \rightarrow 0$ as $s^{\prime} \rightarrow-\infty$, this gives that

$$
w_{a}\left(y^{\prime}, s^{\prime}\right) \rightarrow \kappa \text { as } s^{\prime} \rightarrow-\infty, \text { uniformly on compact sets. }
$$

From parabolic regularity and the continuity of the energy $E\left(w_{a}\right)$, we get from (13) and (15)

$$
E\left(w_{a}\left(s^{\prime}\right)\right) \rightarrow E(\kappa) \text { as } s^{\prime} \rightarrow \pm \infty .
$$

Using the energy identity (7) for $w_{a}$, we conclude that

$$
\int_{-\infty}^{+\infty} d s^{\prime} \int_{\mathbb{R}^{N}}\left|\frac{\partial w_{a}}{\partial s}\left(y^{\prime}, s^{\prime}\right)\right|^{2} \rho d y^{\prime}=E(\kappa)-E(\kappa)=0,
$$

hence $w_{a}$ is just a function of $y^{\prime}$. Using the bound (12) and Proposition 3.1, we see that $w_{a}$ is constant. Using limits (13) and (15), this yields $w_{a} \equiv \kappa$. Consequently, we obtain from (9), (11) and (14):

$$
w(y, s)=w_{a}\left(y^{\prime}, s^{\prime}\right)\left(1+(1-T) e^{s^{\prime}}\right)^{1 /(p-1)}=\kappa\left(1+(1-T) e^{s^{\prime}}\right)^{1 /(p-1)} .
$$


Since we have from $(14) e^{s^{\prime}}=\frac{e^{s-s^{*}}}{1+e^{s-s^{*}}(T-1)}$, we get

$$
w(y, s)=\kappa\left(1+(T-1) e^{s-s^{*}}\right)^{-1 /(p-1)}
$$

hence $w(y, s)=\kappa$ if $T=1$ or $w=\kappa\left(1+e^{s-s_{0}}\right)^{-1 /(p-1)}$ if $T>1$, where $s_{0}=-\log (T-1)+s^{*}$, which is the desired conclusion. This ends the proof of the Liouville Theorem in the nonnegative case.

\section{References}

[FK92] S. Filippas and R.V. Kohn. Refined asymptotics for the blowup of $u_{t}-\Delta u=u^{p}$. Comm. Pure Appl. Math., 45(7):821-869, 1992.

[FKMZ00] C. Fermanian Kammerer, F. Merle, and H. Zaag. Stability of the blow-up profile of non-linear heat equations from the dynamical system point of view. Math. Ann., 317(2):347-387, 2000.

[GK85] Y. Giga and R.V. Kohn. Asymptotically self-similar blow-up of semilinear heat equations. Comm. Pure Appl. Math., 38(3):297-319, 1985.

[GK87] Y. Giga and R. V. Kohn. Characterizing blowup using similarity variables. Indiana Univ. Math. J., 36(1):1-40, 1987.

[GK89] Y. Giga and R. V. Kohn. Nondegeneracy of blowup for semilinear heat equations. Comm. Pure Appl. Math., 42(6):845-884, 1989.

[Kap63] S. Kaplan. On the growth of solutions of quasi-linear parabolic equations. Comm. Pure Appl. Math., 16:305-330, 1963.

[MM00] Y. Martel and F. Merle. A Liouville theorem for the critical generalized Korteweg-de Vries equation. J. Math. Pures Appl. (9), 79(4):339-425, 2000.

[MZ98] F. Merle and H. Zaag. Optimal estimates for blowup rate and behavior for nonlinear heat equations. Comm. Pure Appl. Math., 51(2):139-196, 1998.

[MZ00] F. Merle and H. Zaag. A Liouville theorem for vector-valued nonlinear heat equations and applications. Math. Ann., 316(1):103-137, 2000.

[MZ08] F. Merle and H. Zaag. Openness of the set of non characteristic points and regularity of the blow-up curve for the $1 \mathrm{~d}$ semilinear wave equation. Comm. Math. Phys., 2008. To appear.

[NZ07] N. Nouaili and H. Zaag. A liouville theorem for vector valued nonlinear heat equations with no gradient structure and applications to blow-up. 2007. Submitted.

[Zaa06] H. Zaag. Determination of the curvature of the blow-up set and refined singular behavior for a semilinear heat equation. Duke Math. J., 133(3):499-525, 2006. 
Nejla Nouaili

Département de mathématiques et applications -École Normale supérieure

45 rue d'Ulm 75230 PARIS cedex 05

France

E-mail: Nejla.Nouaili@ens.fr 